\title{
EFFECT of DG Optimizing on overload Transmission line Stability
}

\author{
Ali Abdullah Ali ${ }^{1}$, Ahmed N Abdalla ${ }^{1, *}, R$. ISHAT ${ }^{1}$ \\ ${ }^{1}$ Faculty of Engineering Technology, University Malaysia Pahang, Gambang 26300, Malaysia.
}

\begin{abstract}
Typical steady state studies always treat the peak power demands as the worst case conditions. Periods of light load are also critical in the assessment of the possible state of a power system. While heavy load conditions are generally associated with overload, low voltage and generation deficiency, light load conditions may give rise to over-voltage and undesirable reactive power requirements at generation side. This paper focus on study the effect of DG Optimizing on overload Transmission line Stability. The system dispatch constraints should be taken into account to compensate or varying DG generation output and to enhance the operational performance of power systems. This dispatching operation depends on the change of DG generation and the dispatching strategy. The impact of DG generation uncertainty is limited with the generation dispatching operation and should not be neglected in system analysis.
\end{abstract}

\section{Introduction}

In power system operating and planning studies, the reliability of the system should be assessed and monitored. The uncertainty affects both the long and medium term system planning, and the day-ahead operation. DG power generation has become an increasingly important renewable energy resource due to its ability to produce electric power at varying capacity levels and at varying voltage levels including both distributed generation and dedicated commercial sized plants. Some US state mandates have set a goal of $20 \%$ renewable generation by 2020 [1]. All these bring us to think about how to transmit and distributed and generate the electricity appropriately in order to make us enjoy the electricity more convenient. And what is more, as our construction and production are more and more relay on the electricity in modern time. If we are failed in any part of the electricity production, it will lead to the power outage and a loss of even millions of dollars [2].

One important aspect to analyze is the uncertainty of the power system with DG generation installed. Uncertainty deals with characterizing the possible distribution of the expected variable based on its historical statistical data [3]. The probabilistic power flow (PPF) algorithm has been successfully utilized for calculating the effect of the propagation of data in accuracies through the power flow calculation, thus obtaining a range of values for

\footnotetext{
* Corresponding author: dramaide@ yahoo.com
} 
each output quantity that, to a high degree of probability, bounds the operating conditions of the system [3],[4]. This probabilistic algorithm takes into consideration the uncertainty of the system information and is able to provides tract bounds for the determined variables of the system. As an input this approach requires information regarding the range of all possible system conditions that might been countered as a result of expected uncertainties of loads and other system variables. Assuming that the variable can exist anywhere within a precisely restricted interval, the variable is described to vary ac- cording to some probability distribution. Thus, the system variables can be characterized not by a single number but by a range of values [5]. This correlation is considered and statistically characterized. Besides, the stochastic behavior of PV generation usually does not follow a normal distribution. Due to these characteristics, PV generation can potentially cause various power quality issues in power systems [2]-[6], such as fluctuation of bus voltage magnitudes and line flows, voltage violations and unbalanced power flows. Thus the scheduling for the worst cases cenario is not easily defined. Therefore, the steady state analysis should not just consider the peak power demand cases. In actual system operation, the influence of conventional generation should also be considered, since these resources are essential to balance the power supply and demand, and relieve the uncertainty problem. It is not a realistic assumption to let the slack bus generation balance the change of all the system power injections.

In this paper, introduce analysis the traditional equations to get the power loss and make a induce in mathematic to get a new power loss equation. Then, we use a new analysis way of get the optimal DG. For the Power World, we find this software have a strong function on analysis power flow. We only need to set up the modeling for power system. The result that we could get is clear and direct shown on the line modeling and also for medina an Extra Excel table.

\section{Materials and Method}

\subsection{Distributed Generator (DG)}

It is naturally to propose a method about employing DG in secondary networks to regulate abnormal voltage profile locally. However, obviously, it is not wise that using DG that located at south-east cornerof networks to regulate abnormal voltage happened at north-west corner. Therefore, the issue about how to use DG to regulate meshed secondary networks voltage profile with only local information and communication is studied systematically in this dissertation: firstly, a method about determination of each DG's influence range on secondary network voltage profile and how to group all distributed generators are proposed; then, how to select the proper distributed generators to involve in voltage regulation, and finally, a DG based optimal decentralized voltage regulation for meshed secondary network, which is only based on "local" information and communication for DG working is modeled, simulated and analysed[7].

\subsection{Relationship between DG Output and Voltage Profile}

At the point when DG is incorporated in optional systems, DG impacts voltage profile of each hub with various degrees. This impact can be linearzed by affectability lattice around a specific working purpose of system, which has been utilized as a part of electric force framework investigation for a long time [8]-[10]. Affectability network is acquired from taking after methods [11]:

For any k node in power system, we have 


$$
\tilde{S}_{k}=P_{k}+Q_{k}=\tilde{V}_{k}+\tilde{I}_{k}^{*}
$$

where $\tilde{S}_{k}$ is the complex power flowing through node, $P_{k}$ is the active power flowing through node, $Q_{k}$ is the reactive power flowing through node, $\tilde{V}_{k}$ is the phasor voltage to the ground at node, $\tilde{I}_{k}^{*}$ is the conjugate phasor current flowing into the network at node. From system node admittance matrix, we have

$$
\tilde{I}_{k}=\sum_{m=1}^{n} \tilde{Y}_{k m} \tilde{V}_{m}
$$

where $n$ is the total number of nodes. $\tilde{Y}_{k m}$ is the phasor admittance between node and $\mathrm{m}$ and k. Substitution of $\tilde{I}_{k}$ given by equation (2) in equation (1) yield

$$
P_{k}+Q_{k}=\tilde{V}_{k} \sum_{m=1}^{n}\left(G_{k m}-J B_{k m}\right) \tilde{V}_{m}^{*}
$$

The product of phasor $\tilde{V}_{k}$ and $\tilde{V}_{m}^{*}$ can be expressed as

$$
\begin{aligned}
\tilde{V}_{k} \tilde{V}_{m}^{*} & =\left(V_{k} e^{j \theta_{k}}\right)\left(V_{m} e^{-j \theta_{m}}\right) \\
& =V_{k} V_{m} e^{j\left(\theta_{k}-\theta_{m}\right)}=V_{k} V_{m}\left(\cos \theta_{k m}-j \sin \theta_{k m}\right)
\end{aligned}
$$

where $\theta_{k m}=\theta_{k}-\theta_{m}$

In this way, equation(3)canbere-writtenasfollowingbysubstitutingequation(4) into equation (2.3):

$$
\begin{gathered}
Q_{k}=V_{k} \sum_{m=1}^{n}\left(G_{k m} V_{m} \cos \theta_{k m}+B_{k m} V_{m} \sin \theta_{k m}\right) \\
P_{k}=V_{k} \sum_{m=1}^{n}\left(G_{k m} V_{m} \sin \theta_{k m}-B_{k m} V_{m} \cos \theta_{k m}\right)
\end{gathered}
$$

From equation(5)and(6), $P$ and $Q$ at each bus are functions of voltage magnitude $V$ and angle $\theta$ of all buses. If active power and reactive power at a bus are specified, using $s p$ to denote specified values, load flow equation can be written as following:

$$
\left\{\begin{array}{l}
P_{n}\left(\theta_{1}, \ldots, \theta_{n}, V_{1}, \ldots, V_{n}\right)=P_{n}^{s p} \\
Q_{n}\left(\theta_{1}, \ldots, \theta_{n}, V_{1}, \ldots, V_{n}\right)=Q_{n}^{s p}
\end{array}\right.
$$

Since affectability network is acquired, with starting voltage $V_{0 i}$ and reference voltage $V_{r i}$ at a specific hub $\mathrm{i}$, we can register the generator receptive force era change.

$$
\begin{aligned}
& x_{Q j}=Q_{r j}-Q_{0 j} \\
& x_{P j}=P_{r j}-P_{0 j}
\end{aligned}
$$

where $\mathrm{Q}_{\mathrm{rj}}$ and $\mathrm{Q}_{0 \mathrm{j}}$ are receptive force yield after and before voltage regulation, separately, or the dynamic force era conformity. $P_{r j}$ And $P_{0 j}$ are active power outputs after and before voltage regulation, respectively, at node $\mathrm{j}$ to control voltage from $V_{0 i}$ to $V_{r i}$ of node i using

$$
V_{r i}=V_{0 i}+\Lambda_{i j}^{V Q} x_{Q j}+\Lambda_{i j}^{V P} \cdot x_{P j}
$$




\subsection{DG Dispatching Operation}

In actual applications, realistic dispatching laws are complicated and could cause significant computational problems. Therefore these dispatching laws are complicated for the PPF algorithm to handle. To find a meaningful approach to represent the dispatching laws, a linear model is adopted. The linear model of generation dispatching operationis given by.

$$
\Delta P=T \Delta P_{P V}+\Delta P_{g e n, F}
$$

where $\Delta P_{g e n}$ and $\Delta P_{\text {Wind }}$ are the uncertainty variable vectors of $P_{g e n}$ and $P_{\text {Wind }} T$ is the sensitivity matrix of conventional generation. $T_{i j}$ represents the change in the $i$ th generation for a unity change in the $j^{\text {th }}$ Wind generation. $\Delta P_{g e n,} F$ is the redistribution of the available generation for some generation outage. Under normal conditions (no generator outages), in this research, the outage conditions are excluded. References [12] and [13] considered the outage conditions. This dissertation only considers the dispatching strategy of balancing the Wind generation variation.

Equation (10) shows how the generation sources balance the changes of Wind generation. This model also indicates that the variations of conventional generation sources depend on the variations of the Wind generation and various dispatching strategies. The conventional generation production variations are not in dependent variables and are functions of Wind generation uncertainties. It is expected that systems with very high penetration of Wind resources will require attention to the high ramp rate capabilities of conventional generation resources to balance the Wind generation. For the generators with low ramp rate, the associated element value in the T matrix in (10) is set to zero. The T matrix may be selected as follows, by deciding how a Wind generation change is to be shared among the neighboring generators; by fitting a linear model over areas on able set of conditions as obtained using an optimal power flow program; by using optimal sensitivity equations, which adjust the generations to minimize the cost.

$$
\Delta \mathrm{P}_{\text {gen }}=\mathrm{T} \Delta \mathrm{P}_{\mathrm{PV}}
$$

Since the probabilistic model of Wind generation is known, the probabilistic model of each conventional generation production can be obtained based on the established dispatching model in (10).

\subsection{Transmission line voltage stability}

Lots of indicators of voltage stability which may evaluate its extent and test its seriousness. Such indicators are adopted to confirm the affinity between the working and critical points. It is of use to understand in analysis of voltage stability an established specific working point of the electric system, what's the distance between the point to the instability of voltage. It allows the workers to foresee and use preventive steps to prevent any impending instability of voltage. A few indexes of voltage stability coming from the flow analysis of static power were raised for the system of utility power. The index values underwent a calculation for every line of distribution according to the results of load flow. In a system, the greatest value line was used as the worse line that got special focus to keep the stability of voltage. An LQP (voltage stability index) neglecting the resistance of line was raised by Mohamed. An FVSI (fast voltage stability index) was yielded by Musirin and others [14].The rapid indicator overlooked the difference of angle between voltages at the two ends of a line. An indicator of voltage was expressed by the power through the injection by loading on the local-bus and the power from the rest of buses in a system of [15]. 


\subsection{Proposed method}

Selecting the proper way of voltage stability evaluation is the first move for the development of the supervision plan of stability of voltage. The increase of reactive and active power, which represents the increase of load, and the outage of line are directly and extensively adopted incidents in the researches of voltage stability of power system. At any working point, the active power, by definition, is the extra load supported by the system, prior to being unstable. The steps included in the voltage stability prediction and determination is indicated in Figure 1.

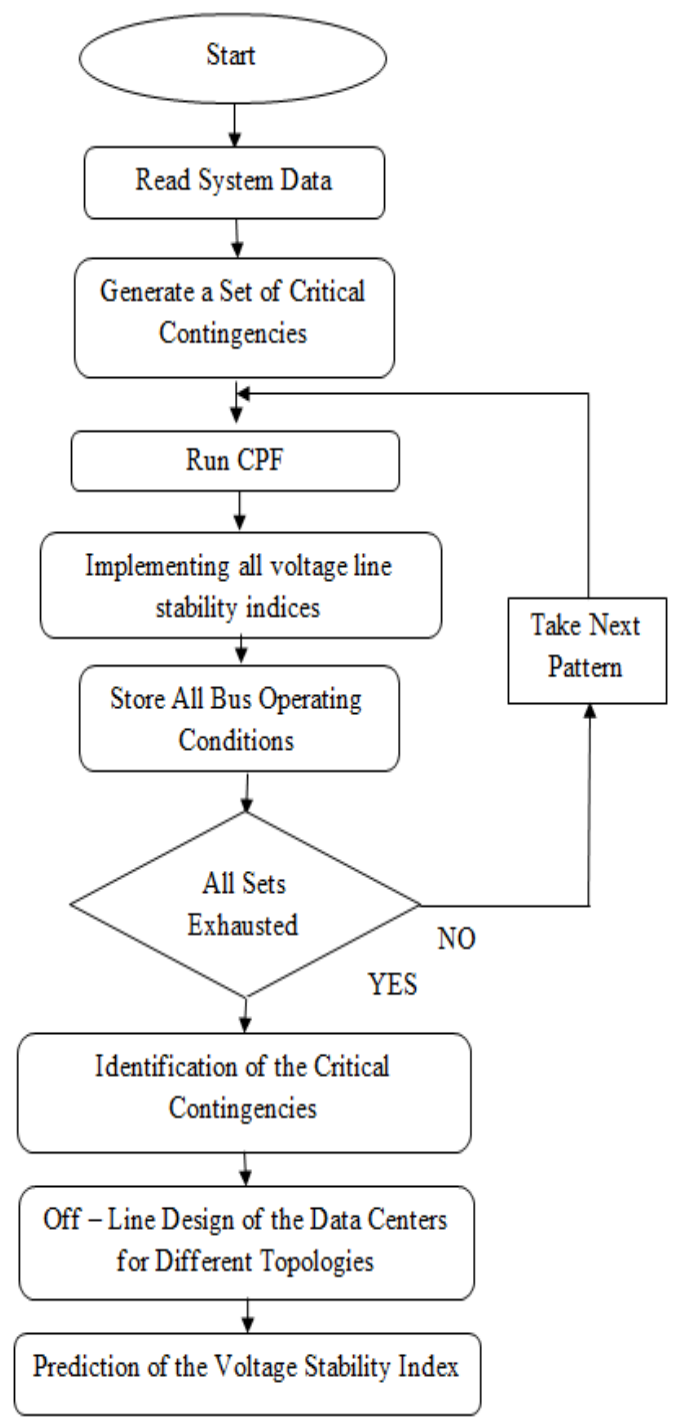

Fig. 1. Voltage stability assessment and prediction overload TL.

The terminal conditions exerted by diverse kinds of nodes in a network of power lead to a non-linear problem, thus the equations of power flow are iteratively resolved by NewtonRaphson or Gauss-Seidel and other technique methods [16]. Such iterations start with predicted voltage magnitude and angle values at overall buses of load and the angle of voltage at buses of generator. Traditional algorithms of power flow in terms of Gauss-Seidel or 
Newton-Raphson techniques cannot converge while the working point reaches the P-V curve`s nose point. Thus, the algorithms that are numerically stable regarded as CPF (Continuation Power Flow) technique have been raised to avoid the issue. The continuous power flow`s fundamental principle is to adopt a scheme of iterative predictor-corrector for tracing the working point on the curve of $\mathrm{P}-\mathrm{V}$ for a specific pattern of load increase [17].

\section{Result and discussion}

The system under study is one of IEEE30 Bus meshed secondary networks The distribution network consists of supplies power from the 6 generator bus and 24 bus network customers and 41 transmission lines [18].

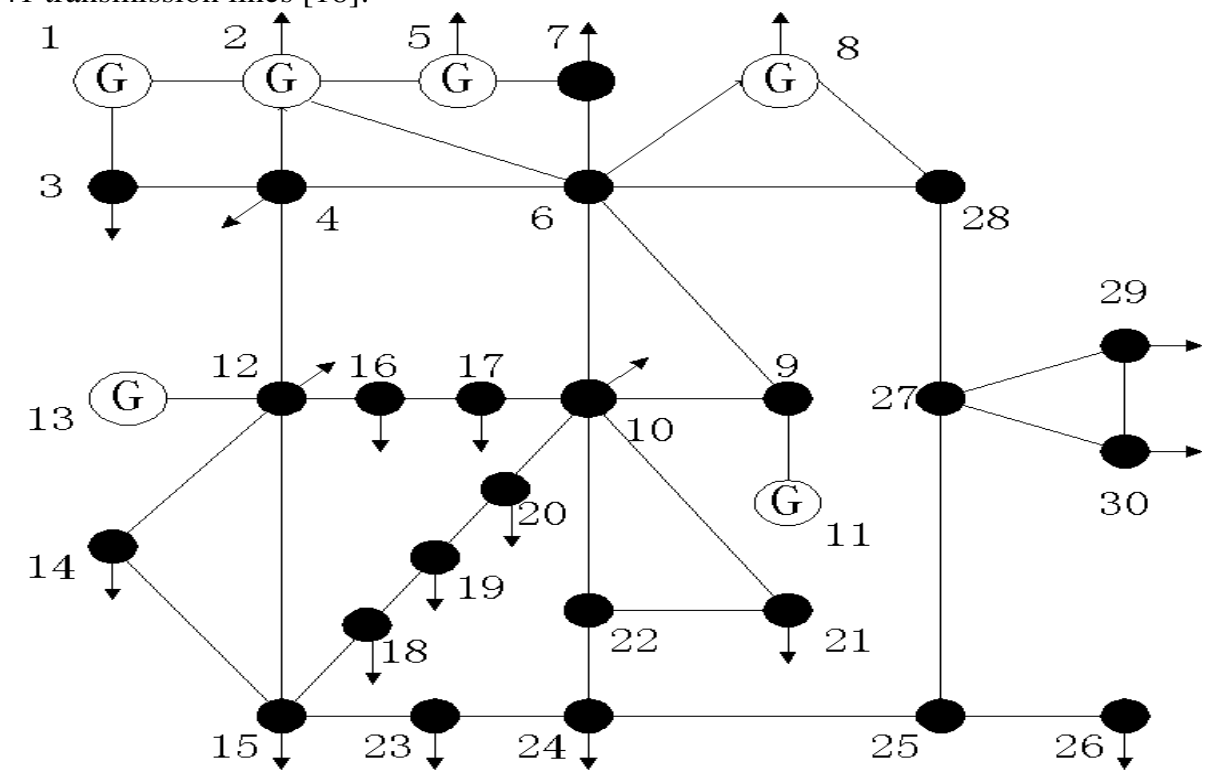

Fig. 2. IEEE 30-bus system.

The simulation result based on two cases to analysis the result for getting the optimal DG power. We could find out that there are two main differences in the two methods:

1.Case 1 the partial derivative, method one do it by inject power $\mathrm{P}_{i}$ while the second method do it by DG power PDG.

2.Case 2 considers more about the p.f. factor problem. It considers all the possible for the PF.

Within the first case, the contingency of load change was performed on the IEEE 30-bus system`s loading buses. While thinking about the three diverse cases of loading, the system`s loading ability was studied. As to static method, the stability indicators of voltage are good for listing the buses and lines that are critical. All indicators are counted in the loading increase that continues before the load flow diverges. Firstly, the reactive and active powers of single bus alters at every loading level at bus 24 and running continuous flow of power to obtain the buses` equivalent voltages. The loading at bus 24 increased gradually varying from the base value $\left(\mathrm{P}_{24} \& \mathrm{Q}_{24}\right)$ to the collapse level of voltage $2.5 *\left(\mathrm{P}_{24} \& \mathrm{Q}_{24}\right)$. Through gradually adding the reactive and active power loads at chosen bus, the experiments were performed and the indicator values as to lines connecting to the bus increased with the greatest values in comparison to the others to show it is the most critical. By observation, the LVSI value 
was highest after FVSI, followed by LQP and $\mathrm{L}_{\mathrm{mn}}$ respectively. The values of all such indicators as to line 31 are shown in Figure 3.

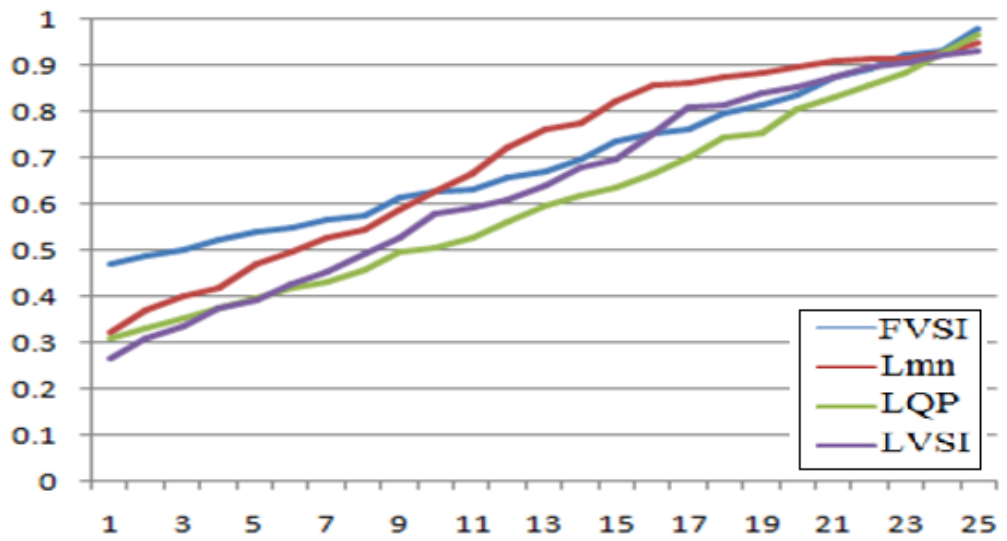

Fig. 3. Effect of increasing (P24) at Bus 24 to FVSI, Lmn, LQP and LVSI evaluated for Line 31.

It may be found that at the greatest loading of active power, the indicator value for Line 31,32 and 33 come to their greatest value. Line 31, at this point, provides the greatest value for all indicators. Therefore, the line comes near to its limit of voltage stability and any forward increase in the loading of active power at bus 25 will lead to the value of FVS1 beyond unity at line 31 because it is in direct connection with bus 24.It indicates that FVSI is the indicator that has the most sensitivity in the indicators raised and line 31 has arrived at its instable state, leading to the collapse of voltage in the overall system. It is expected that bus 24 operates at the condition of loading under this point in order that the safe operation might be kept. The indicator value`s trend at every line in connection to bus 24 while the loading of active power at the bus was improved has been shown in Figure 4. Furthermore, it is indicated that LQP has a greatest post FVSI, and then the LVSI and $\mathrm{L}_{\mathrm{mn}}$ were followed, respectively. All the values of indicator at line 31 were shown in Figure 5.
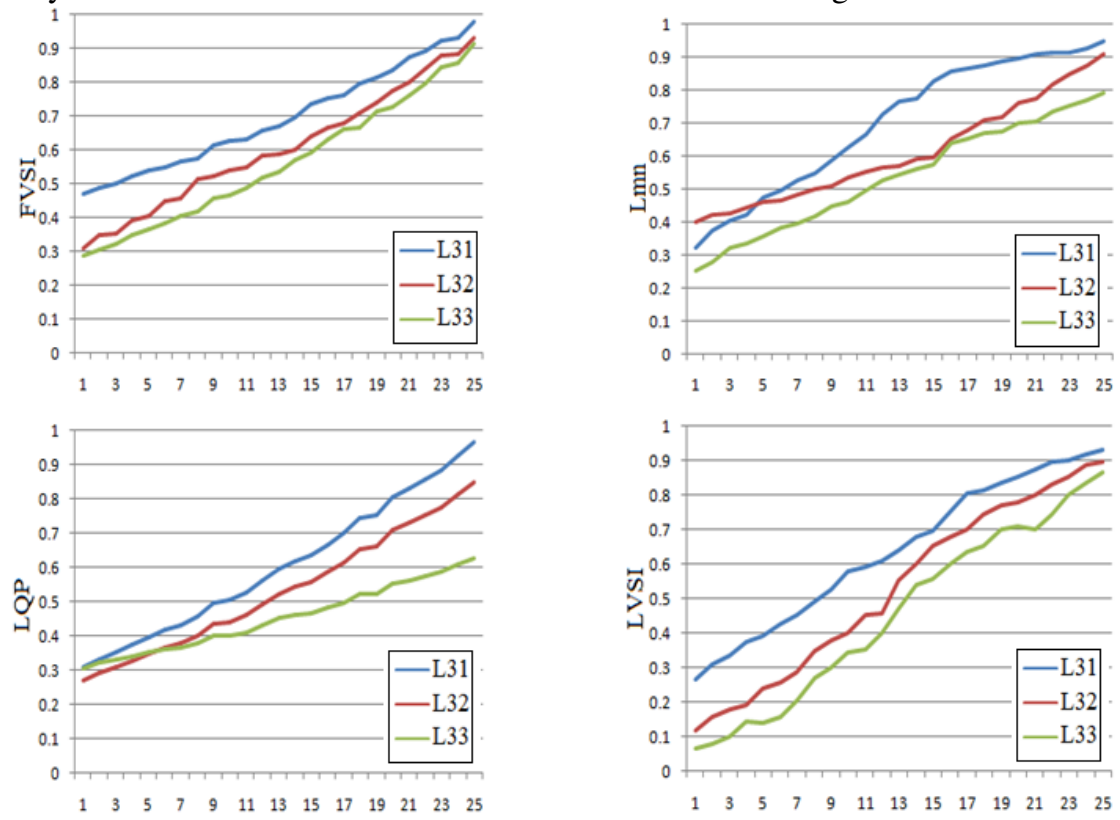

Fig. 4. Effect of increasing (P24) at Bus 24 to FVSI, Lmn, LQP and LVSI evaluated for Lines 31, 32 and 33 . 


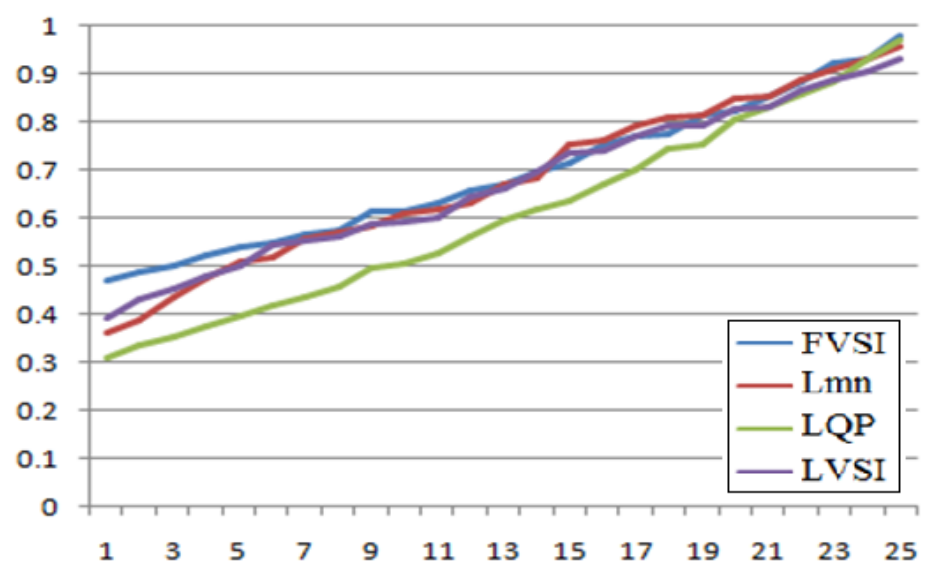

Fig. 5. Effect of increasing (Q24) at Bus 24 to FVSI, Lmn, LQP and LVSI evaluated for Line 31.

For the second case, the contingency line was performed on both lines in connection to the bus 24 within the bus system of IEEE 30 . Both two lines are line 31 with the connection between bus 22 and 24, and line 32 connects between bus 23 and 24. While thinking about the three diverse cases of loading, the system`s loading ability was studied. Under the first condition, the contingency of line was performed on line 31, indicating the disconnection of line 31 , and then the reactive and active power alterations of single bus for every level of loading at bus 24 and the power flow of running continuation to obtain the buses corresponding voltages. The loading at bus 24 increased gradually varying from the base value $\left(\mathrm{P}_{24} \& \mathrm{Q}_{24}\right)$ to the collapse level of voltage $2.5 *\left(\mathrm{P}_{24} \& \mathrm{Q}_{24}\right)$. Through little by little increasing the reactive and active power loading at the chosen bus, and the indicator values as to the rest lines in connection with that bus that increased and possessed greatest values while being in comparison to the rest lines. It shows that the rest lines indicated the situation that is the most critical. It indicates that FVSI is the indicator that has the most sensitivity in the indicators raised and line 32 has arrived at its instable state, leading to the collapse of voltage in the overall system. The indicator tread value at line 33 and 32 in connection to bus 24 was shown in Figure 6. Seen from Figure 6, the indicator values were added correspondingly to the increment of loading. Also, the line 32 has the greatest value for all indicators and thus is considered as the line that is the most sensitive. 

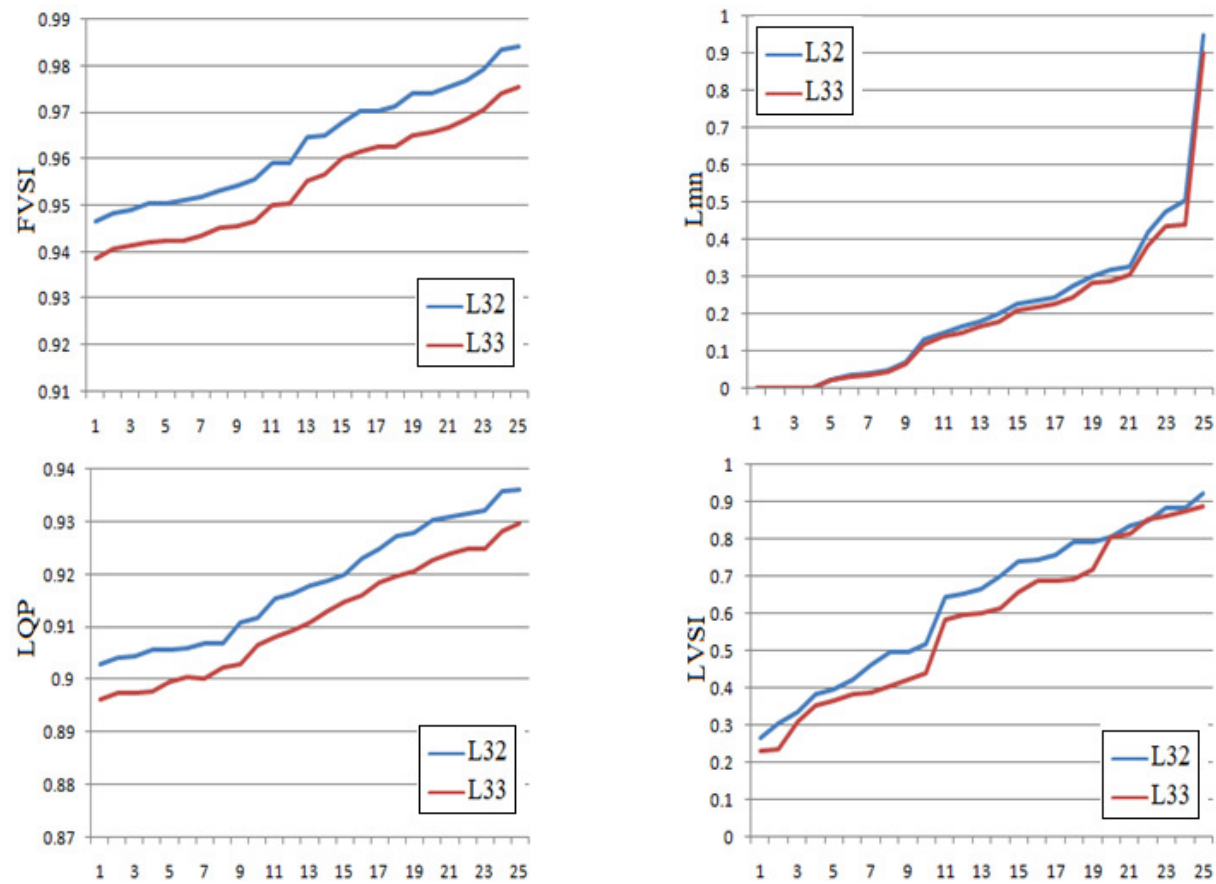

Fig. 6. Line Contingency Effect of Line 31 to FVSI, Lmn, LQP and LVSI evaluated for Lines 32 and 33.

Additionally, the contingency of line was performed on line 32, indicating the disconnection of line 32, and then the reactive and active power alterations of single bus for every level of loading at bus 24 and the power flow of running continuation to obtain the buses` corresponding voltages. The loading at bus 24 increased gradually varying from the base value $\left(\mathrm{P}_{24} \& \mathrm{Q}_{24}\right)$ to the collapse level of voltage $2.5 *\left(\mathrm{P}_{24} \& \mathrm{Q}_{24}\right)$. Through gradually adding the reactive and active power loads at chosen bus, the experiments were performed and the indicator values as to lines connecting to the bus increased with the greatest values in comparison to the others to show it is the most critical. It is indicated that LQP has a greatest post FVSI, and then the LVSI and $\mathrm{L}_{\mathrm{mn}}$ were followed, respectively. The values of all such indicators as to line 31 are shown in Figure 7.

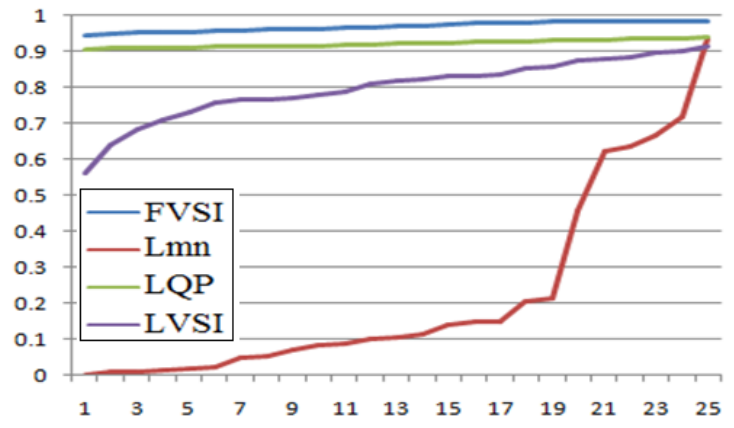

Fig. 7. Line Contingency Effect of Line 32 to FVSI, Lmn, LQP and LVSI evaluated for Line 31. 


\section{Conclusion}

In this paper, the important contributions are presented in the assessment of voltage stability area in order for the protection of transmission line from overloading within the system of electric power. At first, the indicators of voltage stability for some contingency were implemented using a VSA (voltage stability assessment) method; which implemented repeating flow of load, indicator prediction and computation as to the variation in reactive and active power loading at the chosen bus of loading. Results acquired from the researched were therefore derived for the line that is critical and the identification of bus for the comparison between the conventional and the predicted VSA techniques. The simultaneous increase of load for reactive and active power at some load bus was implemented so as for the identification of suitable loading condition prior to a system began for stability loss. Additionally, the comprehensive comparison researches between all indicators have been performed using diverse contingencies and the analysis of feature sensitivity adopted for the improvement of the voltage stability indicators` performance.

\section{References}

1. A. Wang Z., and Alvarado L. Interval Arithmetic in Power Flow Analysis. Proceedings of Power Industry Computer Application conference, 156 - 162 (1991)

2. Rahman S., and Bouzguenda M, IEEE Power Eng. Rev., 9(2): 224 - 230 (2002)

3. Dopazo J.F., Klitin O.A., Sasson A.M, IEEE T. Power Ap. Syst., 94(2): 299 - 309 (1975)

4. Borkowska B. Probabilistic Load Flow, IEEE T. Power Ap. Syst., 93(3): 752-759 (1974)

5. Allan R.N., LeitedaSilva A.M. Burchett R.C., IEEE T. Power Ap. Syst., 100(5): 2539 2546 (2003)

6. Monedero J., Dobon F., Lugo A., Valera P., Osuna R., costaL.A, Marichal G, Photovoltaic Energy Conversion, 2: 2043 - 2045 (2003)

7. Pathomthat, Ramakumar R IEEE Trans. on Energy Conv., 19(4): 764-773 (2004)

8. Ayres H. M., Freitas W., De Almeida M. C., Da Silva L. C. P, IEEE Proc. Gener.Transm.Distrib., 4(4): 495-508 (2010)

9. Farag H.E.Z., El-Saadany E.F., Seethapathy R, IEEE Trans. Smart Grid, 3(1): 271-281 (2012)

10. Zambroni de Souza A.C., Quintana V. H, New technique of network partitioning for voltage collapse margin calculations, IEE Proc.-Gener..Transm..Distrib., 141(6) (1994)

11. Kundur P. Power System Stability and Control, (McGraw-Hill press, 1993)

12. Hu Z., Xifan Wang. A Probabilistic Load Flow Method Considering Branch Out- ages, IEEE T. Power Ap. Syst., 21(2): 507-514 (2006)

13. Allan R. N., Al-Shakarchi M. R. G. Probabilistic techniques in AC load flow analysis, Proceedings of the Institution of Electrical Engineers, 124(2): 154-160 (1976)

14. Ismail, M. and Rahman, T. K. A, IEEE Power Eng. Rev., 22(11): 50-52 (2002)

15. Huang, G., Zhao. Measurement based Voltage Stability Monitoring of Power System, (Power Systems Engineering Research Center, University of Wisconsin, Madison, WI. 2001)

16. Kundur, P. Power System Stability and Control, EPRI Power System Engineering Series, (McGraw Hill, 1994). 
17. Ajjarapu. Computational Techniques for Voltage Stability Assessment and Control: (Springer Verlag, 2006)

18. Sauer, P.W., Pai, M.A. Power system dynamics and stability, Prentice-Hall, (Englewood Cliffs, New Jersey, 1998)

19. Mehdi, O.H., Izzri N., Aballa A.N., Jaber A.S., Shalash N.A., Lafta Y.N, Int. J. Phys. Sci., 6: 7978-7984 (2011)

20. RuhaizadIshak, Azah Mohamed, Ahmed Abdalla N., Mohd ZamriCheWanik, Electric Power Systems Research, 22: 389-398 (2014)

21. Jing Zhang, Gang Li, Ahmed Abd-Alla N., Wen J. Y., Cheng S. J. Theoretical Analysis of the Interaction between Power System Stability Modes with the Normal Forms of Vector Fields, (IEEE, 2006.)

22. Molzahn D. K., Lesieutre B. C., DeMarco C. L. Investigation of non-zero duality gap solutions to a semi definite relaxation of the optimal power flow problem, In Hawaii International Conference on System Sciences, (2014)

23. Ali A., Ahmed N., Ruhaizad B., Aqeel S, Overloaded transmission lines protection based on Intelligent Control Protection Scheme, (MedWell Journals, 2016)

24. Molzahn D., Holzer J., Lesieutre B., DeMarco C, IEEE T. Power Ap. Syst., 28(4): 39873998 (2013) 